\title{
Mind the Gap: Tracking Recommendations to Improve Program Effectiveness
}

\author{
Katherine Johnson \\ Johnson Consulting Group \\ Arkansas Public Service Commission
}

\author{
Scott Reeves \\ The Cadmus Group \\ Nicola Tran \\ The State of Maryland
}

Program evaluations offer essential information regarding all aspects of program operations. But too often, recommendations for program refinement and enhancement are quickly forgotten. This paper illustrates an effective strategy to ensure that these recommendations are taken "off the shelf" and incorporated into future program design and evaluation activities.

This paper describes the process evaluation "best practice" used effectively in several jurisdictions to ensure that program recommendations are well-formulated, strategic, and monitored throughout the evaluation cycle. It begins by describing the types of recommendations that truly make a difference-strategic recommendations that focus on measurable improvements in program delivery-rather than tactical recommendations that target minor adjustments to program implementation.

Several examples from real word evaluations are highlighted in this paper demonstrate the different ways in which this technique can be modified to meet differing program goals. Specific tactics discussed include: creating a recommendation tracking database that organizes each recommendation by topic, sector, and category; classifying the current status of each recommendation; and the reporting interval for updating program recommendations.

Keywords: program evaluation, EM\&V, process evaluation, program tracking

\section{INTRODUCTION}

Program evaluations offer essential information regarding all aspects of program operations. This is especially true for process evaluations, focusing on providing essential and timely feedback regarding current program operations. Therefore, a critical outcome of process evaluations is to provide recommendations that will improve and enhance program operations. However, formulating recommendations that will guide an entire program portfolio or improve overall program operations 
requires thoughtful analysis. Unfortunately, many process evaluations include recommendations as an after-thought. They are hastily written, sometimes repeated verbatim for each evaluated program, and are not carefully tracked over time. The result is that these recommendations are read and maybe overlooked or forgotten, thus diluting the value of the overall process evaluation.

This paper describes how significant recommendations from program or portfolio-wide evaluations are monitored throughout the program cycle. This approach, currently used for statewide evaluations in Arkansas and Maryland, tracks the progress of the key recommendations in each evaluation report.

Monitoring and updating the implementation status of each significant recommendation provides greater transparency regarding program operations and instills confidence that the previous evaluation findings are being incorporated into future program designs. This evaluation approach aligns precisely with the feedback loop developed by The National Action Plan for Energy Efficiency (2007) (see Figure 1).

\section{FIGURE 1 \\ PROGRAM IMPLEMENTATION CYCLE WITH HIGH-LEVEL EVALUATION ACTIVITIES}

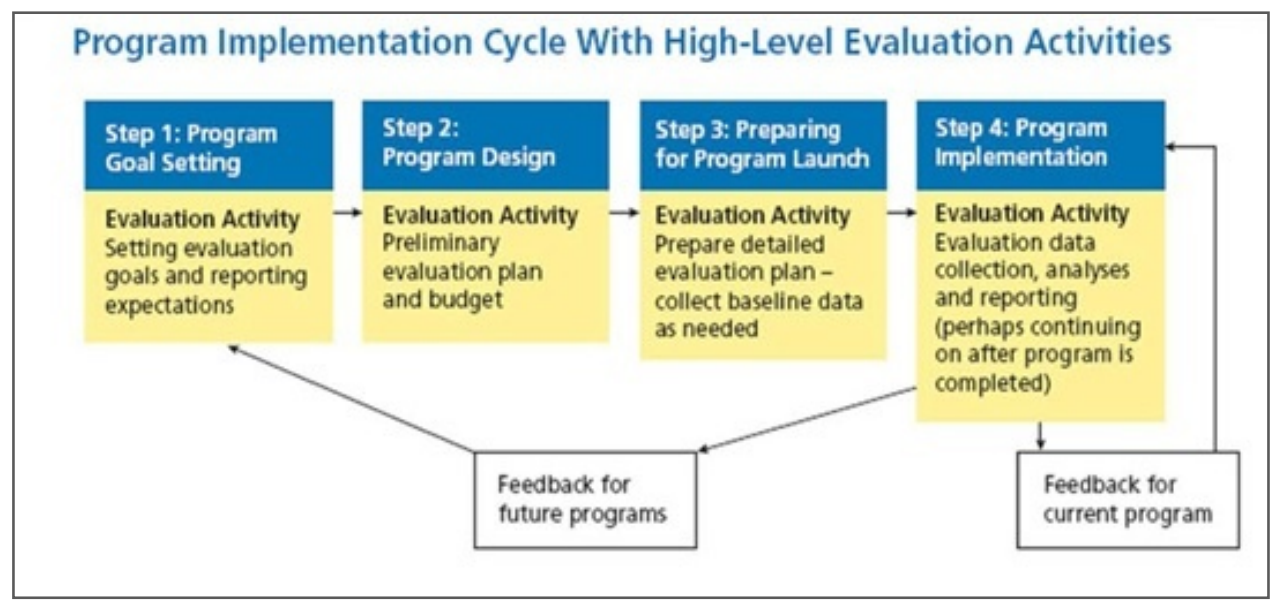

(Source: NAPEE 2007)

As Figure 1 shows, program evaluation is an ongoing process that provides information regarding changes in program direction and adjustments to program goals and objectives over time. Monitoring how the previous evaluation findings have been implemented in current program operations ensures that the critical takeaways of earlier evaluations will be incorporated in the current program design. Thus, tracking the progress of significant recommendations offers a way for evaluators and implementers to assess the effectiveness of these program changes and identify new strategies to consider in evolving energy markets.

\section{ESTABLISHING THE RULES OF THE ROAD}

Unlike impact evaluations, which rely on a set of protocols such as the International Performance Measure and Verification Protocols (IPMVP), only a few jurisdictions have developed specific protocols for conducting process evaluations. In 2012 New York State recognized the importance of establishing Evaluation, Measurement \& Verification (EM\&V) best practices for process evaluations, so it commissioned the development of specific process evaluation protocols.

These protocols were developed based on a comprehensive review of process evaluation "best practices," especially those identified in the California Protocols (2005) but were also expanded to include input from leading process evaluators through a peer review. These guidelines also specifically required periodic reporting of the implementation status of recommendations made in earlier program evaluations.

In 2013, the New York State process evaluation protocols were modified and expanded in several other jurisdictions, including Arkansas. In Arkansas, all EM\&V protocols are incorporated into the current 
Technical Reference Manual (TRM), which provides guidance on various evaluation topics. Other jurisdictions, including Colorado, Idaho, Maryland, and Missouri, have referenced these process evaluation protocols as part of the overall guidance that third-party evaluation contractors should incorporate in their evaluations.

While the process evaluation protocols provide direction on conducting all types of process evaluation activities, this paper focuses on developing actionable recommendations: those improvements that can be implemented during the program cycle. The second critical element of this paper describes how the progress made towards implementing each recommendation is tracked throughout the evaluation cycle.

In Protocol C, Volume 1 of the Arkansas TRM, the protocols require the following:

\section{PROTOCOL C: Process Evaluation Guidance}

Provide a progress report for each recommendation for program improvement made in previously conducted evaluations. For each evaluation recommendation, the report should indicate whether the recommendation has been accepted and implemented, rejected, or is still under consideration. If the recommendation is rejected, an explanation of the reason for rejection should be provided. If a recommendation is still under consideration, then an explanation should be provided for the steps underway to reach an implementation decision for that recommendation;

Any recommendations should be strategic in nature, that is designed to enhance overall program operations or efficiency. The evaluators may also provide tactical suggestions on ways to refine specific program elements, such as refining the marketing messaging or increasing contractor training sessions. These tactical suggestions do not need to be reported or tracked over time. Only strategic recommendations that provide actionable guidance for the program staff and/or implementer must be tracked and reported for every evaluation.

(Source: Arkansas Technical Reference Manual, Version 8.0; August 31, 2018, p. 25)

Requiring the program evaluators to track the disposition of each recommendation ensures that these recommendations will not simply "remain on the shelf."

This protocol provides specific guidance regarding the type of recommendations that should be tracked. The focus is on tracking "strategic" recommendations that will lead to lasting program improvements. Short-term suggestions are viewed a "tactical" recommendations and do not require tracking. Examples of the differences between these types of recommendations are illustrated next.

A "strategic recommendation" would be to increase the marketing and outreach activities to include contractor outreach to increase overall program participation. A "tactical recommendation" would be suggesting running an advertising promotion in March of each year. Strategic recommendations emphasize ways to affect the outcome of the program or portfolio, while tactical recommendations focus on short-term changes. Tactical recommendations are sometimes described as "action plans" and are intended to provide immediate but often short-term results. For this reason, tactical recommendations do not require tracking as they will likely either be implemented or discarded before the subsequent program evaluation.

\section{PRACTICAL APPLICATIONS}

The seven Investor-Owned Utilities (IOUs) in Arkansas conducted independent third-party evaluations that complied with the requirements and methodologies described in the EM\&V protocols. These protocols, which are contained in Volume 1 of its Technical Reference Manual (TRM), include specific guidance on how to track and report the implementation status of the strategic recommendations from each evaluation report. Evaluation activities are conducted annually for each of these utilities. The key findings and recommendations from these evaluations are then reviewed and summarized by the Independent Evaluation Monitor (IEM) in its annual report to the Arkansas Public Service Commission.

Table 1 summarizes the disposition of the previous recommendations that have been made and considered over seven evaluation periods. These recommendations were developed by the two selected 
implementation contractors, and each year, the progress made regarding implementing these recommendations is reported in the annual EM\&V report. The two evaluators developed specific categories to describe each previous recommendation's implementation status, as described in Table 1.

TABLE 1

\section{CATEGORIES FOR TRACKING RECOMMENDATIONS}

\begin{tabular}{|l|c|}
\hline Status of Previous Recommendations (2011-2017) & Total \\
\hline Completed & 803 \\
\hline In Progress & 185 \\
\hline Incomplete & 47 \\
\hline Not Applicable & 50 \\
\hline Reviewed/Rejected & 97 \\
\hline Problem Persists/Not Yet Addressed & 4 \\
\hline Under Consideration & 34 \\
\hline
\end{tabular}

However, these definitions have evolved to reflect changes in program planning. For example, one utility decided to defer implementing any future program changes until the next program cycle, which meant that several recommendations "persisted" or were not yet addressed until the new program filing incorporated the recommended changes in program design.

\section{ARKANSAS EM\&V TRACKING RESULTS}

Figure 2 summarizes the total number of recommendations during seven evaluation cycles for the Arkansas IOUs. As this figure shows, there was a significantly large number of recommendations provided in 2012 for two reasons. First, the evaluators counted each recommendation for each program which led to duplication. Secondly, a majority of these recommendations were, in fact, tactical suggestions that did not address strategic goals. As this figure illustrates, once the evaluators eliminated unnecessary repetition and focused on providing actionable and strategic guidance, the total number of recommendations decreased sharply in subsequent years.

FIGURE 2

TOTAL NUMBER RECOMMENDATIONS REPORTED ANNUALLY FROM 2011-2017

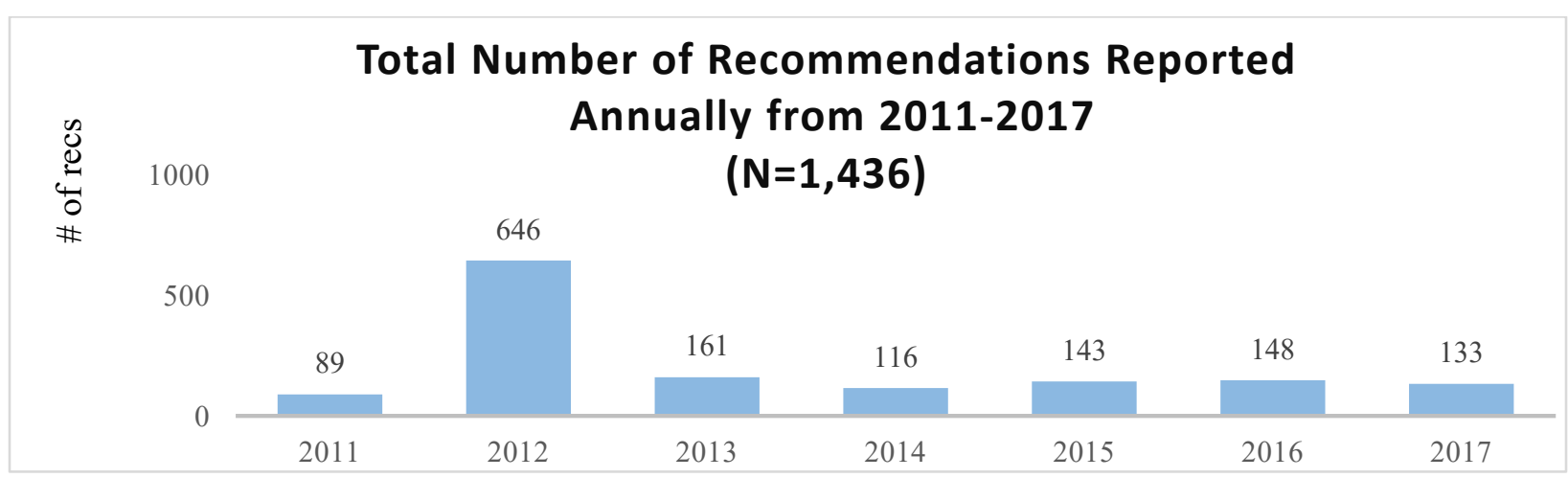

(Source: Summary of the IEM's Annual EM\&V Reports 2011-2017)

Each year, the recommendations were further divided into categories (i.e., process or impact) (see Figure 3). 
FIGURE 3

SUMMARY OF TOTAL NUMBER OF PROGRAM RECOMMENDATIONS BY CATEGORY

FOR PROGRAM YEARS 2011-2017

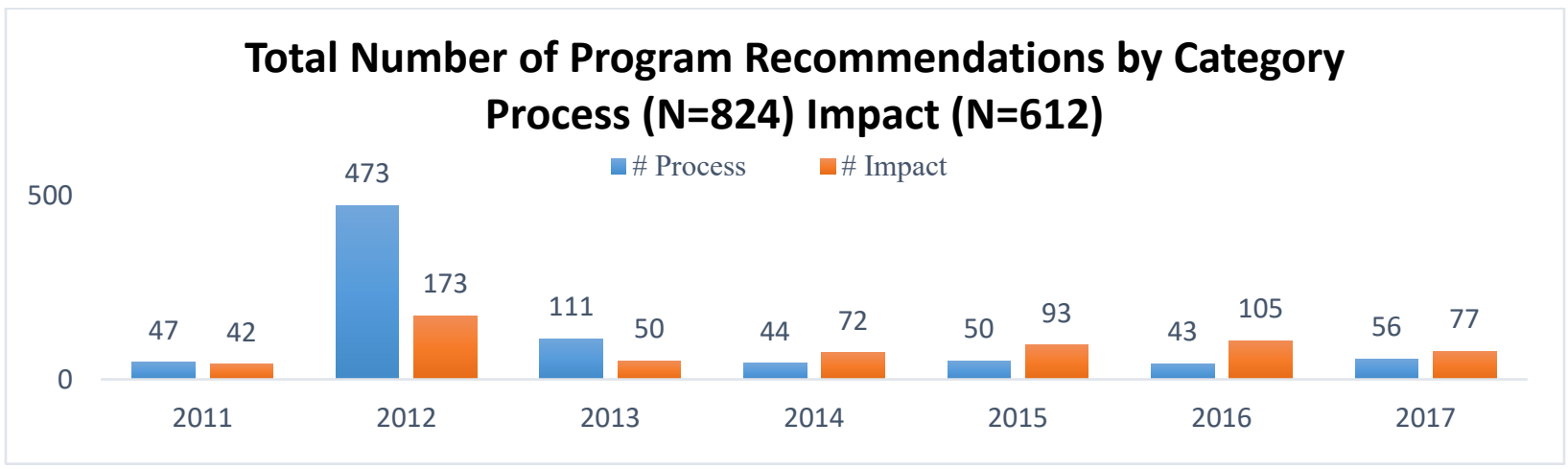

(Source: Summary of the IEM's Annual EM\&V Reports 2011-2017)

Of note, the number of recommendations from process evaluations was significantly higher compared to impact recommendations. This was due, in part, to a misunderstanding of the difference between recommendations compared to suggestions. For example, in 2012, one evaluator provided a total of 302 process recommendations. But most recommendations did not meet the "actionable" criterion, which meant that they did not have to be tracked over time. As this process evolved and only "actionable recommendations" were tracked, the number became much more manageable for the evaluators to monitor over time.

As Figure 4 shows, two-thirds of the recommendations were addressed by the utilities or program implementers during the program cycle. In contrast, a few utilities rejected the recommendations (8\%) for various reasons, including changes in program design, market conditions, or program operations.

FIGURE 4

DISPOSITION OF RECOMMENDATIONS FROM PY2011-PY2017

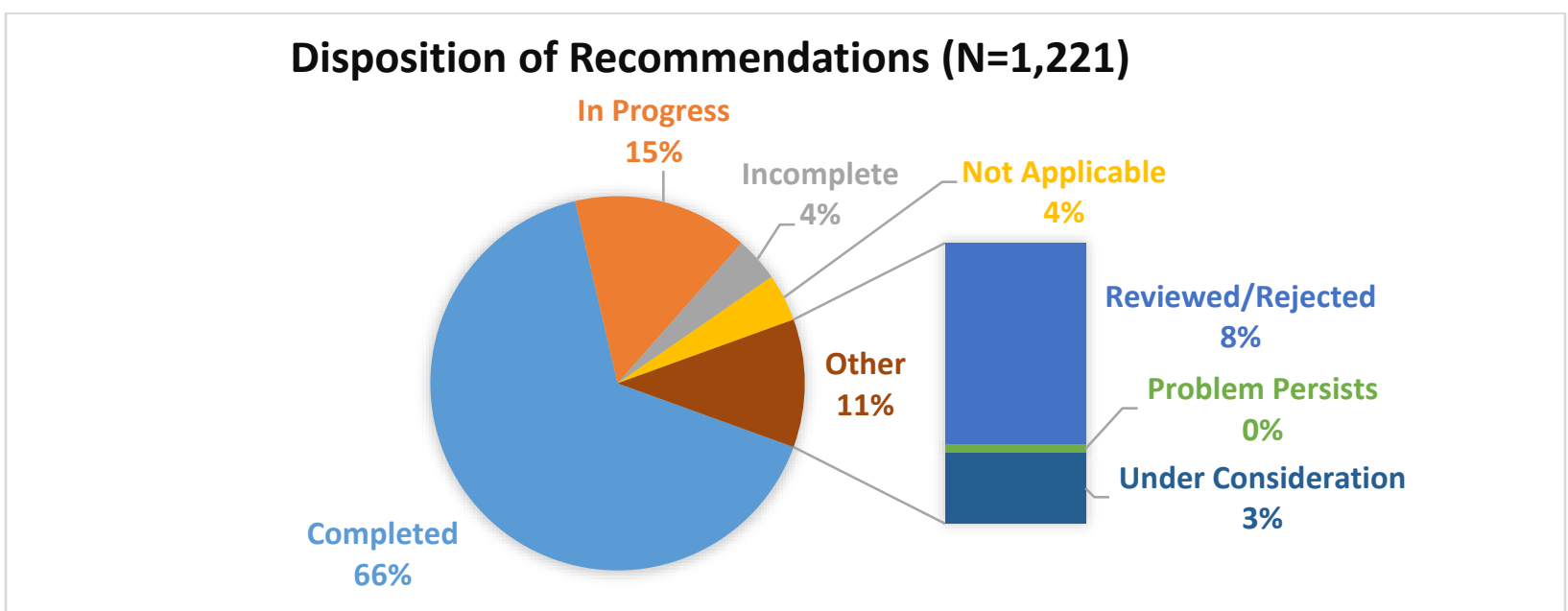

(Source: Summary of the IEM's Annual EM\&V Reports 2011-2017)

\section{RECOMMENDATION TRACKING IN ARKANSAS}

The recommendations are first divided by category (i.e., process or impact). Then, they are further divided into topic areas. As illustrated in Figures 5 and 6, this approach helps pinpoint those specific areas 
that require program improvement across the entire energy efficiency program portfolio within the evaluation period.

FIGURE 5

EXAMPLE OF TRACKING IMPACT RECOMMENDATIONS BY TOPIC AREA

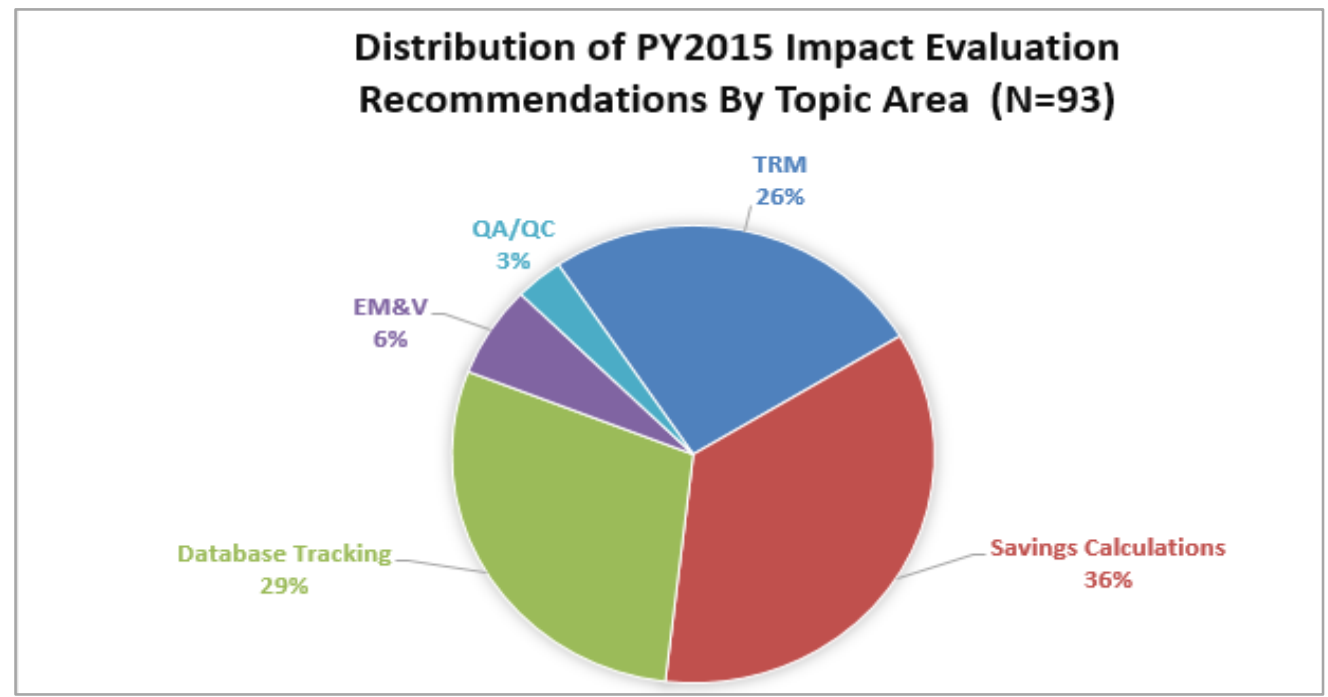

(Source: IEM Annual Report PY2015, p. vi)

FIGURE 6

EXAMPLE OF TRACKING PROCESS EVALUATION RECOMMENDATIONS BY TOPIC AREA

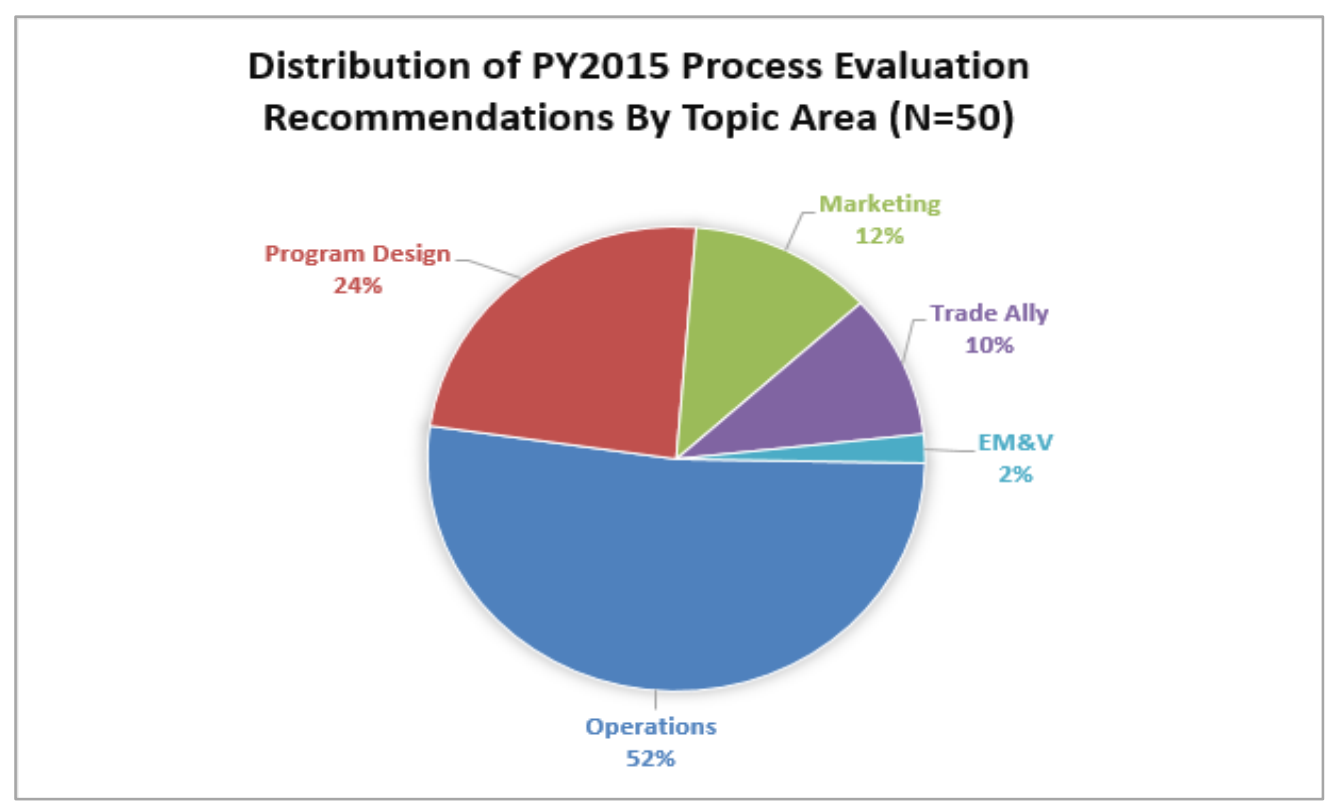

(Source: IEM Annual Report PY2015, p. vi)

Since these evaluations are conducted statewide, the IEM summarizes the number of recommendations by utility. This approach also makes it easy to understand which topics need to be addressed by each utility 
or by fuel type (i.e., electric or gas). This analytical technique also adds another level of transparency to the evaluations.

\section{FIGURE 7 \\ EXAMPLE OF TRACKING PROCESS RECOMMENDATIONS BY ENERGY ORGANIZATION}

\begin{tabular}{|l|c|c|c|c|c|}
\hline Energy Organization & Operations & Program Design & Marketing & Trade Ally & EM\&V \\
\hline AOG & 1 & 2 & 2 & & \\
\hline AOG-OG\&E & 1 & & & & \\
\hline AWP & 1 & & & & \\
\hline BHE & 1 & 1 & & 1 & \\
\hline CenterPoint & 6 & 4 & & 1 & \\
\hline EAI & 9 & 2 & 1 & 1 & \\
\hline Empire & & 1 & & 1 & \\
\hline OG\&E & 3 & 1 & 1 & & \\
\hline SWEPCO & 4 & 1 & 2 & 1 & 1 \\
\hline Total & 26 & 12 & 6 & 5 & 1 \\
\hline
\end{tabular}

(Source: IEM Annual Report PY2015, pp. vii-viii)

But the practicality of tracking recommendations is not always as straightforward as it seems. Even though the evaluators have specific protocols to follow, recommendation tracking has been "hit or miss" during the past seven evaluations. Sometimes the evaluators provided more complete updates regarding the status of each recommendation, while in other years, these summaries fell short. The following excerpts from the previous IEM Annual Reports illustrate these inconsistencies.

IEM Annual Report Excerpt 2012

Industry best practices dictate that the process evaluations begin by assessing the disposition of the previous process evaluations recommendations. Unfortunately, all the evaluations fell short in this area. Therefore, this is a significant area for improvement for all evaluators to pursue in subsequent process evaluation activities going forward. (IEM Annual Report PY20123 EM\&V Report, p. 67).

IEM Annual Report Excerpt 2015

The utilities have considered all of the previous recommendations and implemented more than half of them. The evaluators reported the status of the previous recommendations, in accordance with the Process Evaluation Protocol C. Overall, most of these recommendations $(n=118)$ have been implemented (53\%) while a few are still in progress (22\%). (IEM Annual Report PY2015 EM\&V Report, p. v)

IEM Annual Report, 2017

The reporting on the status of all of the previous recommendations was incomplete for four of the six reports in this evaluation year. (IEM Annual Report PY2017 EM\&V p. 71)

To ensure more thorough and consistent reporting, the evaluators and the IEM worked together to refine the process evaluation protocols and requirements for tracking the progress of recommendations. This process has also been clarified in the process evaluation protocols since 2013 . 
For example, it is now explicitly stated that program recommendations only have to be tracked through the program cycle. This clarification was made so that the recommendations will align with the current three-year program cycle and funding period. This modification also simplifies tracking the implementation status of previous recommendations, as some older recommendations may no longer be relevant if the program design or objectives have changed. In addition, the evaluators are better at identifying the types or categories of recommendations to track-focusing on those with strategic value to enhance the overall program or portfolio rather than tracking specific suggestions for tactical improvement.

Tracking recommendations provide valuable feedback to all parties. The recommendations are developed by the evaluators and then reviewed in each evaluation period with utilities and program implementers in subsequent years. Stakeholders and public commission staff also monitor progress over time and are assured that these recommendations will be reviewed and considered carefully, thereby fulfilling a primary objective of process evaluations.

\section{RECOMMENDATIONS TRACKING IN MARYLAND}

Recommendations tracking has also been incorporated into the annual evaluation activities that are completed for the Department of Housing and Community Development's (DHCD) two low-income weatherization programs: The Low Income Energy Efficiency Program (LIEEP) and the Multifamily Energy Efficiency and Housing Affordability (MEEHA) Program.

A key outcome from the PY2016 process evaluation was to develop a Recommendations Tracker. The evaluators reviewed the 98 recommendations from the following program evaluation reports and memoranda:

- DHCD MEEHA Property Manager Interviews Memo (Cadmus 2018)

- DHCD LIEEP On-Site Assessment Memo (Cadmus 2017)

- LIEEP Evaluation Verification Report (Itron 2017)

- DHCD LIEEP Evaluation Report (Cadmus 2017)

- MEEHA Impact and Process Evaluation (GDS 2015)

- LIEEP: Summary of Verified Program Savings and Recommendations (Itron 2014)

- LIEEP: Impact and Process Evaluation Final Report (GDS 2014)

Developing the recommendations tracker required several iterations to make it a useful tool. Due to delays in evaluations and staff turnover, it was important for current program and public service staff to first understand the status, if any, of the previous recommendations that had been made but not tracked. To consolidate all previous recommendations and identify those that still may apply to current program operations, the first step was to summarize all of the previous evaluation recommendations by category. The next step was to streamline the tracker by removing those recommendations that were no longer relevant given the new program configuration. The evaluator then worked with the DHCD staff to eliminate redundancies. Table 2 summarizes the number of recommendations that are now tracked by program and category. 
TABLE 2

SUMMARY OF RECOMMENDATIONS TRACKER FOR DHCD'S LIEEP AND MEEHA PROGRAMS

\begin{tabular}{|c|c|c|c|}
\hline Program & Category & $\begin{array}{c}\text { Total \# of } \\
\text { Recommendations }\end{array}$ & $\begin{array}{c}\text { Total \% of } \\
\text { Recommendations }\end{array}$ \\
\hline \multirow{13}{*}{ LIEEP } & Communications & 2 & $2 \%$ \\
\hline & Customer Follow-up & 1 & $1 \%$ \\
\hline & Customer Satisfaction & 2 & $2 \%$ \\
\hline & Customer Targeting & 1 & $1 \%$ \\
\hline & Database Tracking & 13 & $13 \%$ \\
\hline & Energy Education & 3 & $3 \%$ \\
\hline & Energy Savings & 0 & $0 \%$ \\
\hline & Evaluation & 15 & $15 \%$ \\
\hline & Marketing \& Outreach & 8 & $8 \%$ \\
\hline & Program Design & 8 & $8 \%$ \\
\hline & Program Operations & 18 & $18 \%$ \\
\hline & $\mathrm{QA} / \mathrm{QC}$ & 3 & $3 \%$ \\
\hline & $\mathrm{TRM}$ & 2 & $2 \%$ \\
\hline \multirow{13}{*}{ MEEHA } & Communications & 2 & $2 \%$ \\
\hline & Customer Follow-up & 0 & $0 \%$ \\
\hline & Customer Satisfaction & 0 & $0 \%$ \\
\hline & Customer Targeting & 0 & $0 \%$ \\
\hline & Database Tracking & 5 & $5 \%$ \\
\hline & Energy Education & 0 & $0 \%$ \\
\hline & Energy Savings & 1 & $1 \%$ \\
\hline & Evaluation & 2 & $2 \%$ \\
\hline & Marketing \& Outreach & 0 & $0 \%$ \\
\hline & Program Design & 5 & $5 \%$ \\
\hline & Program Operations & 1 & $1 \%$ \\
\hline & $\mathrm{QA} / \mathrm{QC}$ & 1 & $1 \%$ \\
\hline & TRM & 0 & $0 \%$ \\
\hline \multirow{13}{*}{ LIEEP/MEEHA } & Communications & 0 & $0 \%$ \\
\hline & Customer Follow-up & 0 & $0 \%$ \\
\hline & Customer Satisfaction & 0 & $0 \%$ \\
\hline & Customer Targeting & 0 & $0 \%$ \\
\hline & Database Tracking & 0 & $0 \%$ \\
\hline & Energy Education & 0 & $0 \%$ \\
\hline & Energy Savings & 1 & $1 \%$ \\
\hline & Evaluation & 4 & $4 \%$ \\
\hline & Marketing \& Outreach & 0 & $0 \%$ \\
\hline & Program Design & 0 & $0 \%$ \\
\hline & Program Operations & 0 & $0 \%$ \\
\hline & $\mathrm{QA} / \mathrm{QC}$ & 0 & $0 \%$ \\
\hline & TRM & 0 & $0 \%$ \\
\hline Total & & 98 & $100 \%$ \\
\hline
\end{tabular}

(Source: DHCD LIEEP and MEEHA Recommendations Tracker for PY2016-PY2017) 
For the PY2017 program evaluation, DHCD staff provided an update of each recommendation using the following rubric:
1-Completed
2-In Progress/Delayed
3-Partially Complete
4-Rejected
5-Not Applicable

Table 3 illustrates these results.

TABLE 3

EXAMPLE OF RECOMMENDATIONS TRACKING -DHCD'S LIEEP PROGRAM

\begin{tabular}{|c|c|c|c|c|c|c|c|}
\hline \multirow[b]{2}{*}{ Type } & \multicolumn{5}{|c|}{ Current Status } & \multirow[b]{2}{*}{$\begin{array}{c}\text { Total \# of } \\
\text { Recommendations }\end{array}$} & \multirow[b]{2}{*}{$\begin{array}{c}\text { Total \% of } \\
\text { Recommendations }\end{array}$} \\
\hline & Complete & $\begin{array}{c}\text { In } \\
\text { Progress } \\
/ \\
\text { Delayed }\end{array}$ & $\begin{array}{l}\text { Partially } \\
\text { Complete }\end{array}$ & Rejected & $\begin{array}{c}\text { Not } \\
\text { Applicable }\end{array}$ & & \\
\hline Impact & 2 & 6 & 10 & 0 & 0 & 45 & $46 \%$ \\
\hline Process & 10 & 4 & 14 & 0 & 0 & 49 & $50 \%$ \\
\hline Best Practices & 0 & 2 & 1 & 0 & 0 & 4 & $4 \%$ \\
\hline Total & 12 & 0 & 0 & 0 & 0 & 98 & $100 \%$ \\
\hline
\end{tabular}

(Source: DHCD Recommendations Tracker PY2016-PY2017)

This approach led to the successful update of the status of recommendations during each annual evaluation, documenting the changes that have occurred within each program each year and providing a greater level of transparency for the interested stakeholders, including the utilities, EM\&V oversight staff, and Commission staff.

\section{CONCLUSIONS}

This paper illustrates how tracking recommendations can help to preserve the value of previous program evaluations, increase overall transparency, and ensure that all appropriate recommendations are considered and implemented through the program evaluation cycle. The key benefits of recommendations tracking include the ability to:

- Provide another way to identify the effectiveness of program operations over time

- Pinpoint areas for improvement in meaningful ways

- Increase overall accountability and transparency during the evaluation period

- Integrate recommendations into new program designs and;

- Create a vital record to document program achievements

Using a recommendations tracker ensures that these program recommendations fulfill the over-arching objective of program evaluation- to provide guidance for program operations and input for program design, as summarized in the NAPEE feedback loop. 


\section{REFERENCES}

DHCD Spreadsheet Recommendations Tracker PY2-16-PY2017. (2018, September).

Environmental Protection Agency. (2007, November). The National Action Plan for Energy Efficiency: Model Energy Efficiency Program Impact Evaluation Guide, Washington D.C. Retrieved from https://www.epa.gov/sites/production/files/2015-08/documents/evaluation_guide.pdf

Johnson Consulting, Apex Analytics, Eisenberg Energy, Mesa Point Energy, Michaels Energy, Rouleau Consulting. (2012, June 1). Independent Evaluation Monitor EM\&V Annual Report, PY2011 EM\&V Report.

Johnson Consulting, Apex Analytics, Eisenberg Energy, Mesa Point Energy, Michaels Energy, Rouleau Consulting. (2013, May 31). Independent Evaluation Monitor EM\&V Annual Report, PY2012 EM\&V Report.

Johnson Consulting, Apex Analytics, Eisenberg Energy, Mesa Point Energy, Michaels Energy, Rouleau Consulting. (2014, May 30). Independent Evaluation Monitor EM\&V Annual Report, PY2013 EM\&V Report.

Johnson Consulting, Apex Analytics, Eisenberg Energy, Mesa Point Energy, Michaels Energy, Rouleau Consulting. (2015, June 1). Independent Evaluation Monitor EM\&V Annual Report, PY2014 EM\&V Report.

Johnson Consulting, Apex Analytics, Eisenberg Energy, Mesa Point Energy, Michaels Energy, Rouleau Consulting. (2016, August 1). Independent Evaluation Monitor EM\&V Annual Report, PY2015 EM\&V Report.

Johnson Consulting, Apex Analytics, Eisenberg Energy, Mesa Point Energy, Michaels Energy, Rouleau Consulting. (2017, July 7). The Forward Curve, Independent Evaluation Monitor EM\&V Annual Report, PY2016 EM\&V Report.

Johnson Consulting, Apex Analytics, Eisenberg Energy, Mesa Point Energy, Michaels Energy, Rouleau Consulting. (2018, July 6). Independent Evaluation Monitor EM\&V Annual Report, PY2017 EM\&V Report.

Johnson Consulting, Apex Analytics, Eisenberg Energy, Mesa Point Energy, Michaels Energy, Rouleau Consulting. (2017, August 31). Technical Reference Manual, Version 7.0. Volume 1: EM\&V Protocols, Submitted to the Arkansas Public Service Commission.

Johnson, K., \& Eisenberg, G. (2012, January 6). New York State Process Evaluation Protocols. A Supplement to the New York State Evaluation Guidelines Updated 2012.

The Cadmus Group. (2017, July 3). DHCD Low-Income Energy Efficiency Program Evaluation Report. Maryland Department of Housing and Community Development. 